\title{
The long noncoding RNA Tug1 connects metabolic changes with kidney disease in podocytes
}

\author{
Szu Yuan Li ${ }^{1,2}$ and Katalin Susztak \\ ${ }^{1}$ Renal-Electrolyte and Hypertension Division, Department of Medicine, Perelman School of Medicine, University of Pennsylvania, Philadelphia, Pennsylvania, USA. ${ }^{2}$ Division of Nephrology, Department of \\ Medicine, Taipei Veterans General Hospital and School of Medicine, National Yang-Ming University, Taipei, Taiwan.
}

\begin{abstract}
An increasing amount of evidence suggests that metabolic alterations play a key role in chronic kidney disease (CKD) pathogenesis. In this issue of the $J C l$, Long et al. report that the long noncoding RNA (IncRNA) taurine-upregulated 1 (Tug1) contributes to CKD development. The authors show that Tug1 regulates mitochondrial function in podocytes by epigenetic targeting of expression of the transcription factor PPAR $\gamma$ coactivator $1 \alpha$ (PGC-1 $\alpha$, encoded by Ppargc1a). Transgenic overexpression of Tug1 specifically in podocytes ameliorated diabetesinduced CKD in mice. Together, these results highlight an important connection between IncRNA-mediated metabolic alterations in podocytes and kidney disease development.
\end{abstract}

\section{IncRNAs: not just noise}

While less than $2 \%$ of the genome is translated into proteins, recent advances in RNA-sequencing (RNA-seq) technologies have revealed that approximately three-quarters of the mammalian genome is transcribed into RNA. The majority of these transcripts have no protein-coding potential (1); however, several roles and families of these nonprotein-coding RNA species have been deciphered. Long noncoding RNAs (lncRNAs) encompass a large and diverse class of transcribed RNA molecules that are longer than 200 nucleotides and do not encode proteins. Initially, lncRNAs were considered transcriptional noise. However, evidence is accumulating that the majority of noncoding RNAs are functional (2). Whole-genome sequencing has detected approximately 30,000 lncRNA transcripts to date, and studies have revealed that these lncRNAs are species specific, overall poorly conserved, and expressed at low levels. Unfortunately, fewer than 300 lncRNAs have been functionally annotated in the Long Noncoding RNA Database (3). IncRNAs have several modes of action but are generally considered important transcriptional regulators. Enhancer RNAs are considered a subtype of IncRNA that are transcribed from the enhancer region and physically involved in looping the enhancer and promoter regions to regulate transcription. Some lncRNAs can act through chromatin remodeling (mostly by altering the polycomb-repressive complex 2 [PRC2]) to achieve transcriptional regulation. Other lncRNAs interfere with splicing, RNA stability, or translation (Figure 1).

The Cancer Genome Project has highlighted differential expression of lncRNAs in multiple cancer types, and lncRNA signatures are emerging as novel diagnostic cancer biomarkers, as most cancer types are characterized by dysregulated expression of several hundred specific lncRNA species. Conversely, little is known about lncRNA functions and regulations in noncancerous tissues. In this issue, Long et

\section{Related Article: p. 4205}

Conflict of interest: The Susztak laboratory has received research support from Boehringer Ingelheim, Biogen, ONO Pharmaceutical Company, and Celgene for projects that are not related to this work. K. Susztak received consulting fees from Merck, GlaxoSmithKline, and Janssen.

Reference information: / Clin Invest. 2016;126(11):4072-4075. doi:10.1172/JCI90828. al. (4) performed RNA-seq on glomeruli from diabetic mice, specifically focusing on identifying changes in lncRNA levels compared with those in nondiabetic animals, and found that taurine-upregulated 1 (Tug1) levels were consistently reduced in diabetic mouse glomeruli. While most lncRNAs show species-specific expression and regulation, Tug1 expression was found to be evolutionally conserved and was also decreased in human diabetic kidney samples (4). Long at al. localized the Tug1-specific changes to the podocytes and therefore set out to further study the role of Tug1 in podocytes and in development. Using animal and cell culture models, Long and colleagues demonstrated that Tug1 regulates expression in podocytes of the transcription coactivator PPAR $\gamma$ coactivator $1 \alpha$ (PGC- $1 \alpha)$, which regulates genes involved in energy metabolism. According to their data, Tug1 acts as a bridge between the 400-kb upstream enhancer and promoter of Pgcla, thereby regulating PGC- $1 \alpha$ expression (4). In a high-glucose environment, Tug1 expression was decreased, resulting in decreased Pgcla transcription in podocytes.

\section{Podocytes in diabetes}

Podocytes are specialized glomerular epithelial cells that form a layer of the filtration barrier in the kidney. Podocytes are terminally differentiated with limited capacity to renew. In animal models, podocyte injury and loss occurs early during the course of diabetes (5). Low podocyte numbers are a strong predictor of future functional decline in human subjects with either type 1 or type 2 diabetes; therefore, the identification of early metabolic changes in podocytes is thought to be critical for understanding the pathogenesis of diabetic kidney disease (6).

Recent studies indicate that podocytes are insulin sensitive and utilize glucose as their energy source. Podocyte-specific insulin receptor-KO mice develop severe 


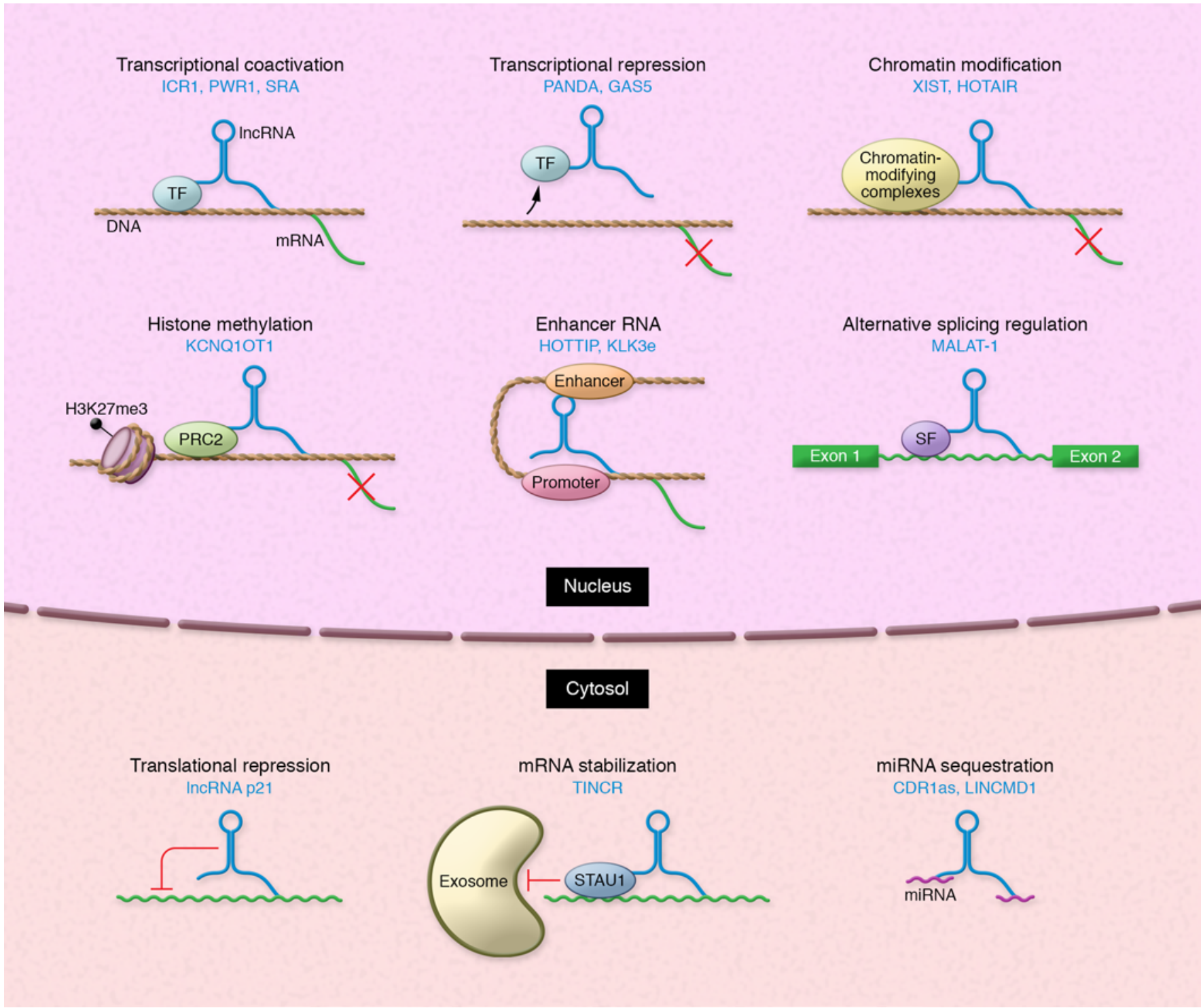

Figure 1. IncRNAs can interfere with gene function at multiple levels. Studies have described a range of mechanisms by which IncRNAs regulate their targets. In the cell nucleus, IncRNAs can regulate transcription by recruiting chromatin-modifying complexes and transcriptional factors to silence or enhance target gene expression. Additionally, some IncRNAs regulate gene expression by influencing pre-mRNA splicing. In the cytosol, IncRNAs can regulate mRNA expression by altering mRNA stability, mRNA translation, or by competing for microRNA binding. ICR1,Interfering Crick RNA 1; PWR1, Promoting Watson RNA1; SRA, Steroid receptor RNA Activator PANDA, P21 associated ncRNA DNA damage activated, PANDA antisense RNA GAS5, Growth arrest-specific 5; TF, Transcription factor; XIST, X-inactive specific transcript; HOTAIR, HOX transcript antisense RNA; KCNQ10T1, KCNQ1 overlapping transcript 1; PRC2, Polycomb Repressive Complex 2; HOTTIP, HOXA transcript at the distal tip; KLK3e, Kallikrein-related peptidase 3 eRNA; SF, Splicing factor; STAU1, Staufen Double-Stranded RNA Binding Protein 1; TINCR, Tissue Differentiation-Inducing Non-Protein Coding RNA; CDR1as, Cerebellar degeneration-related protein 1 Antisense RNA; LINCMD1, long intergenic non-protein coding RNA, muscle differentiation 1.

glomerulosclerosis, even in the absence of hyperglycemia (7). The effect of insulin receptor deletion appears to be independent of glucose uptake, as mice with podocyte-specific deletion of glucose transporter type 4 (Glut4) do not develop similar changes (8). Insulin-induced activation of AKT acts as an important regulator of cytoskeletal changes and prosurvival factor in podocytes and might explain the strong phenotype develop- ment in the podocyte-specific insulin receptor-KO mice (9). Insulin also regulates mitochondrial function via the transcription factor FOXO (10).

In addition to glucose utilization, proper mitochondrial function is essential for podocyte health. Patients with mutations in genes encoding different mitochondrial enzymes, such COQ2, COQ6, COQ10, and aarF domain-containing kinase 4 (ADCK4), present with podocy- topathy and chronic kidney disease (CKD) (11). In animal models, genetic deletion of mitochondrial transcription factors such as Kruppel-like factor 6 (KLF6) specifically from podocytes not only causes mitochondrial defects but is sufficient to induce glomerulosclerosis and kidney failure (12).

In diabetic animal models, both quantitative and qualitative mitochondrial defects have been described and thought to contribute to CKD develop- 


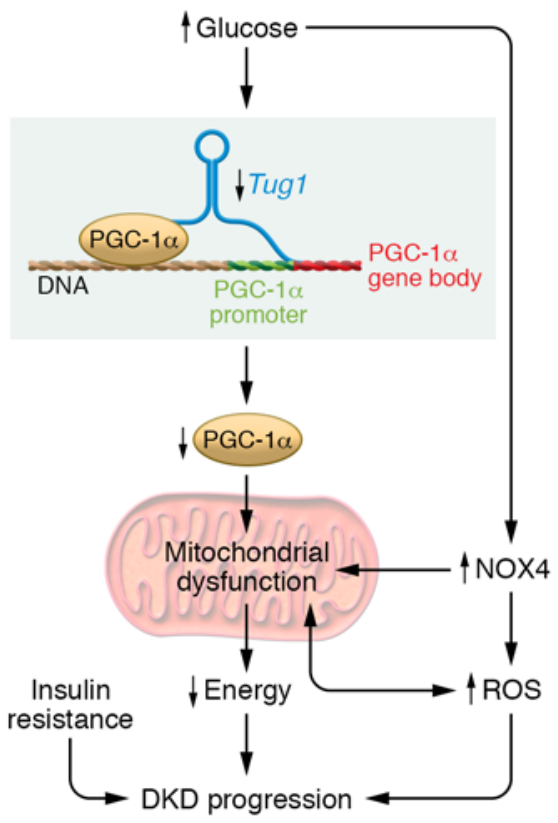

Figure 2. Proper mitochondrial function is essential for podocyte health in diabetes. Tug1 expression is decreased in diabetic podocytes. Tug1 regulates PCC-1 $\alpha$ expression by epigenetic targeting. Decreased PGC- $1 \alpha$ levels results in structural and functional mitochondrial changes. Impaired mitochondrial function will cause energy depletion, increased ROS generation, and, ultimately, the development of diabetic kidney disease. DKD, diabetic kidney disease; NOX4, NAPDH oxidase 4.

ment. Healthy mitochondria are believed to be elongated and filamentous. In contrast, diabetic podocytes are characterized by small, fragmented mitochondria (13), indicating a potential fission problem. The kinase Rho-associated coiled coil-containing protein kinase 1 (ROCK1) has emerged as an important mediator of mitochondrial fission in diabetes mellitus (13). High glucose levels activate ROCK1, which then recruits the GTPase dynamin-related protein 1 (DRP1) from the cytoplasm to the mitochondrial outer membrane, thereby inducing mitochondrial fragmentation (13). In diabetic mice, Rock1 deletion can restore mitochondrial morphology and function as well as attenuate albuminuria.

Podocytes isolated from diabetic mouse models show alterations in mitochondrial gene expression (14), including genes encoding upstream transcription factors, such as PGC- $1 \alpha$ and transcription factor A, mitochondrial (TFAM), that are key regulators of mitochondrial biogene- sis and gene expression. PGC-1 $\alpha$ expression is lower in diabetic glomeruli, and the level of PGC- $1 \alpha$ correlates with structural and functional damage. The expression and regulation of PGC- $1 \alpha$ is complex and includes transcriptional and posttranscriptional changes. Epigenetic regulation of PGC- $1 \alpha$ has also been described, including cytosine methylation changes in addition to the Tug1-induced changes reported by Long et al.

\section{Tug1 does it in vivo}

Like most other lncRNAs, the functional role of TUG1 has been poorly understood. TUG1 expression is increased in most cancer types, and knockdown of TUG1 in cultured cancer cells has been shown to suppress cell proliferation, invasion, and colony formation (15). Given that Tug1 has multiple targets and can interfere with several steps in the process of carcinogenesis, it has been proposed as a therapeutic target. Despite overall poor conservation between species, several lncRNAs are ultraconserved. Metastasis-associated lung adenocarcinoma transcript 1 (MALAT1) is conserved between human and mice. MALAT1 expression is increased in human cancer samples. MALAT-1-deficient mice have no phenotype at baseline; however, these animals are resistant to breast and lung cancer development. Furthermore, MALAT1-targeting antisense oligonucleotides have shown efficacy in a preclinical breast cancer model, indicating the therapeutic potential for targeting this lncRNA (16). Like MALAT1, Tug1 appears to be evolutionarily conserved between mice and humans and similarly regulated in both species.

To explore the function of Tug1 in podocytes and diabetic kidney disease, Long et al. generated transgenic mice that overexpress Tug1 specifically in podocytes (4). These animals appeared healthy at baseline and were crossed with $d b / d b$ mice, which serve as a model of diabetic nephropathy. Podocyte-specific overexpression of Tug1 attenuated diabetesinduced ROS formation, albuminuria, and histopathological changes. Long and colleagues propose that the beneficial effect of Tug1 is fully mediated by restoration of PGC- $1 \alpha$ expression, which restores mitochondrial bioenergetics in podocytes. Together, these results highlight a previously unknown role for Tug1.

\section{The bigger picture}

The study by Long et al. (4) is fully consistent with recent studies indicating that mitochondrial health and PGC- $1 \alpha$ play a critical role in the kidney. For example, gene expression studies have identified mitochondrial transcripts, including those associated with fatty acid oxidation, as one of the most differentially expressed gene groups in CKD (17). Transgenic expression of Pgc1a in renal tubule cells protects mice from both acute and chronic kidney injury $(17,18)$. The work by Long et al. now shows that the PGC- $1 \alpha$ mitochondrial axis is equally important in podocytes (Figure 2) and provides novel mechanistic insight into PGC-1 $\alpha$ regulation. By identifying a IncRNA with podocyte-specific function, this study is an important first step in understanding how these noncoding RNAs regulate kidney function. Future studies aimed at understanding the role of lncRNAs and metabolic changes in CKD have the potential to provide important insight and possible therapeutic targets for treating kidney disease.

\section{Acknowledgments}

This work was supported by National Institutes of Health grants DK076077 and DK087635

Address correspondence to: Katalin Susztak, Renal-Electrolyte and Hypertension Division, Department of Medicine, University of Pennsylvania, Perelman School of Medicine, 415 Curie Blvd., 405 Clinical Research Building, Philadelphia, Pennsylvania 19104, USA. Phone: 215.898.2009; E-mail:ksusztak@mail.med.upenn.edu.

1. Djebali S, et al. Landscape of transcription in human cells. Nature. 2012;489(7414):101-108.

2. Mercer TR, Dinger ME, Mattick JS. Long non-coding RNAs: insights into functions. Nat Rev Genet. 2009;10(3):155-159.

3. Quek XC, et al. lncRNAdb v2.0: expanding the reference database for functional long noncoding RNAs. Nucleic Acids Res. 2015;43(Database issue):D168-D173.

4. Long J, et al. Long noncoding RNA Tug1 regulates mitochondrial bioenergetics in diabetic nephropathy. JClin Invest. 2016;126(11):4205-4218.

5 . Susztak K, et al. Molecular profiling of diabetic mouse kidney reveals novel genes linked to glomerular disease. Diabetes. 2004;53(3):784-794.

6. Reidy K, Kang HM, Hostetter T, Susztak K. Molecular mechanisms of diabetic kidney disease. J Clin Invest. 2014;124(6):2333-2340.

7. Welsh GI, et al. Insulin signaling to the glomer- 
ular podocyte is critical for normal kidney function. Cell Metab. 2010;12(4):329-340.

8. Guzman J, et al. Podocyte-specific GLUT4-deficient mice have fewer and larger podocytes and are protected from diabetic nephropathy. Diabetes. 2014;63(2):701-714.

9. Canaud G, et al. AKT2 is essential to maintain podocyte viability and function during chronic kidney disease. Nat Med. 2013;19(10):1288-1296.

10. Cheng Z, et al. Foxo1 integrates insulin signaling with mitochondrial function in the liver. Nat Med. 2009;15(11):1307-1311.

11. Malaga-Dieguez L, Susztak K. ADCK4 “reen- ergizes" nephrotic syndrome. J Clin Invest. 2013;123(12):4996-4999.

12. Mallipattu SK, et al. Krüppel-like factor 6 regulates mitochondrial function in the kidney. JClin Invest. 2015;125(3):1347-1361.

13. Wang $\mathrm{W}$, et al. Mitochondrial fission triggered by hyperglycemia is mediated by ROCK1 activation in podocytes and endothelial cells. Cell Metab. 2012;15(2):186-200.

14. Fu J, et al. Comparison of glomerular and podocyte mRNA profiles in streptozotocin-induced diabetes. J Am Soc Nephrol. 2016;27(4):1006-1014.

15. Li Z, Shen J, Chan MT, Wu WK. TUG1: a pivotal oncogenic long non-coding RNA of human cancers. Cell Prolif. 2016;49(4):471-475.

16. Zuckerman JE, Davis ME. Clinical experiences with systemically administered siRNA-based therapeutics in cancer. Nat Rev Drug Discov. 2015;14(12):843-856.

17. Kang HM, et al. Defective fatty acid oxidation in renal tubular epithelial cells has a key role in kidney fibrosis development. Nat Med. 2015;21(1):37-46.

18. Tran M, et al. PGC-1 $\alpha$ promotes recovery after acute kidney injury during systemic inflammation in mice. JClin Invest. 2011;121(10):4003-4014. 